\title{
Highly Efficient Dissolution of Lignin by Eutectic Molecular Liquids
}

Qiaoling Liu, ${ }^{\mathrm{a}}$ Hongyu Mou, ${ }^{\mathrm{b}}$ Wenjun Chen, ${ }^{\mathrm{b}}$ Xinhui Zhao, ${ }^{\mathrm{b}}$ Haitao $\mathrm{Yu},{ }^{\mathrm{a}}$ Zhimin Xue, ${ }^{* \mathrm{a}}$ and Tiancheng $\mathrm{Mu}^{* \mathrm{~b}}$

${ }^{a}$ Beijing Key Laboratory of Lignocellulosic Chemistry, College of Materials Science and Technology, Beijing Forestry University, Beijing 100083, China.Email: zmxue@bjfu.edu.cn.

${ }^{b}$ Department of Chemistry, Renmin University of China, Beijing 100872, China. Email: tcmu@ruc.edu.cn.

\section{Solubility determination}

The solubility of lignin in the prepared EMLs was determined through a two-step process.

First, the examined EML ( $c a .1 .0 \mathrm{~g}$ ) was added into a flask of $25 \mathrm{~mL}$ at the desired temperatures (i.e., 30, 50, 70, and $90^{\circ} \mathrm{C}$ ). When the temperature was constant, samples of lignin (i.e., DAL, EHL, SL, and OL) were continually added with an increment size of $c a .15 \mathrm{mg}$ until the added lignin could not be dissolved in $2 \mathrm{~h}$. From this step, the lignin solubility with higher potential deviations could be obtained.

Subsequently, lignin (with a mass of $\mathrm{m}_{1} \mathrm{~g}$ ) was weighed out on an electronic balance (with the precision of $0.0001 \mathrm{~g}$ ). Lignin with a $90 \%$ amount of the first-step achieved solubility was initially added into a glass flask of $25 \mathrm{~mL}$ containing the desired EML (with a mass of $\mathrm{m}_{3} \mathrm{~g}$, ca. $1.0 \mathrm{~g}$ ) at different temperatures (i.e., 30, 50, 70, and $\left.90^{\circ} \mathrm{C}\right)$. After the added lignin was dissolved completely, samples of lignin were continually added with an increment size of $c a$. $5 \mathrm{mg}$ until the lignin could not be dissolved in $2 \mathrm{~h}$. Finally, the amount of the remained lignin (mass of $\mathrm{m}_{2} \mathrm{~g}$ ) could be achieved on the electronic balance. The solubility of lignin in specific EMLs could be achieved based on the following formula:

$$
\text { Solubility }=\frac{\left.\mathrm{m}_{1} \text { (initial lignin }\right)-\mathrm{m}_{2}(\text { remained lignin })}{\left.\mathrm{m}_{1} \text { (initial lignin }\right)-\mathrm{m}_{2}(\text { remained lignin })+\mathrm{m}_{3}(\mathrm{EML})} \times 100 \%
$$

Herein, it should be pointed out that some modifications would be done for the cases with the solubilities of $<5 \mathrm{wt} \%$ or $>45 \mathrm{wt} \%$ (from the first step). For the cases with the solubilities of $<5 \mathrm{wt} \%$, the amount of the weighted out lignin was changed into $c a .0 .1 \mathrm{~g}$, and the increment size of lignin became ca. $2 \mathrm{mg}$. Furthermore, for the cases with the solubilities of $>45 \mathrm{wt} \%$, the amount of the weighted out lignin was changed into $c a .1 .3 \mathrm{~g}$. 
For each experiment, the second step was repeated twice, and an average value was provided as the lignin solubility with the standard deviations (Table S4-S7). For the cases with a solubility of $<5 \mathrm{wt} \%$, the second step was only conducted once and did not consider the standard deviations because of the too low solubility. Meanwhile, the two steps were both repeated if too large difference was detected between the first and the second step, with the purpose to ensure the accuracy of the achieved solubility.

\section{Solvatochromic parameters measurement.}

A brief description of the procedure of the determination solvatochromic parameters $(\alpha, \beta$ and $\left.\pi^{*}\right)$ is given below.

Firstly, the dyes were dissolved in methanol with a concentration of $1.0 \times 10^{-3} \mathrm{~mol} / \mathrm{L}$. Then, 50 $\mu \mathrm{L}$ dye solution was added into a centrifuge tube. After that, the tube was heated at $313 \mathrm{~K}$ under vacuum for $30 \mathrm{~min}$ to remove the methanol. Subsequently, the desired EML (2.5 g) was added into the tube and well mixed with the dye. Finally, the mixed liquid was put into a quartz cell with a light path length of $1.0 \mathrm{~cm}$ and the absorption spectra were recorded (Varian Cary 50 UV-vis spectrophotometer) at $298 \mathrm{~K}$.

$\pi^{*}$ and $\alpha$ can be calculated by the following equation: ${ }^{1,2}$

$$
\begin{gathered}
\pi^{*}=\left[19.839-v_{N R}\right] / 2.9912 \\
\alpha=\left[19.9657-1.0241 \times \pi^{*}-v_{N R}\right] / 1.6078
\end{gathered}
$$

$v_{N R}=1 /\left[\lambda_{(\mathrm{NR}) \max } \times 10^{-4}\right]$, where $\lambda_{(\mathrm{NR}) \max }$ represented the maximum absorption wavelength of Nile red.

$\beta$ value can be calculated by the following equation: ${ }^{3}$

$$
\beta=11.134-3580 / \lambda_{(\mathrm{NH} 2) \max }-1.125 \times \pi^{*}
$$

$\lambda_{(\mathrm{NH}) \max }$ was the maximum absorption wavelength of 4-nitroaniline. 
Table S1. The temperature for the formation of the used DBU-based EMLs.

\begin{tabular}{|c|c|c|}
\hline Entry & EML & Temperature $\left({ }^{\circ} \mathrm{C}\right)$ \\
\hline 1 & DBU-LA-2:1 & 25 \\
\hline 2 & DBU-LA-1:1 & 25 \\
\hline 3 & DBU-LA-1:2 & 25 \\
\hline 4 & DBU-LA-1:3 & 25 \\
\hline 5 & DBU-PEG-2:1 & 25 \\
\hline 6 & DBU-PEG-1:1 & 25 \\
\hline 7 & DBU-PEG-1:2 & 25 \\
\hline 8 & DBU-PEG-1:3 & 25 \\
\hline 9 & DBU-CPL-2:1 & 25 \\
\hline 10 & DBU-CPL-1:1 & 45 \\
\hline 11 & DBU-CPL-1:2 & 50 \\
\hline 12 & DBU-CPL-1:3 & 60 \\
\hline 13 & DBU-NMTU-2:1 & 30 \\
\hline 14 & DBU-NMTU-1:1 & 50 \\
\hline 15 & DBU-BZA-2:1 & 25 \\
\hline 16 & DBU-BZA-1:1 & 50 \\
\hline 17 & DBU-PCA-2:1 & 25 \\
\hline
\end{tabular}


Table S2. The viscosity $(\eta)$ of the prepared EMLs at different temperatures. ${ }^{\text {a }}$

\begin{tabular}{|c|c|c|c|c|c|}
\hline \multirow{2}{*}{ Entry } & \multirow{2}{*}{ EML } & \multicolumn{4}{|c|}{ Viscosity $(\mathrm{MPa} \cdot \mathrm{s})$} \\
\hline & & $25^{\circ} \mathrm{C}$ & $35^{\circ} \mathrm{C}$ & $45^{\circ} \mathrm{C}$ & $55^{\circ} \mathrm{C}$ \\
\hline 1 & DBU-LA-2:1 & 356.35 & 153.27 & 76.14 & 43.49 \\
\hline 2 & DBU-LA-1:1 & 2720.90 & 908.86 & 365.24 & 173.43 \\
\hline 3 & DBU-LA-1:2 & 3096.30 & 1053.00 & 426.36 & 206.28 \\
\hline 4 & DBU-LA-1:3 & 403.17 & 181.22 & 92.59 & 52.49 \\
\hline 5 & DBU-PEG-2:1 & 65.14 & 38.40 & 24.16 & 16.43 \\
\hline 6 & DBU-PEG-1:1 & 77.38 & 23.14 & 14.47 & 9.78 \\
\hline 7 & DBU-PEG-1:2 & 69.00 & 41.24 & 26.70 & 18.39 \\
\hline 8 & DBU-PEG-1:3 & 62.50 & 37.88 & 24.72 & 17.00 \\
\hline 9 & DBU-CPL-2:1 & 26.02 & 17.90 & 11.80 & 6.02 \\
\hline 10 & DBU-CPL-1:1 & 41.28 & 23.14 & 14.47 & 9.78 \\
\hline 11 & DBU-CPL-1:2 & -- & -- & -- & -- \\
\hline 12 & DBU-CPL-1:3 & -- & -- & -- & -- \\
\hline 13 & DBU-NMTU-2:1 & 446.80 & 147.03 & 62.36 & 31.56 \\
\hline 14 & DBU-NMTU-1:1 & 7147.20 & 1984.3 & 474.95 & 156.93 \\
\hline 15 & DBU-BZA-2:1 & 262.62 & 102.38 & 48.17 & 26.55 \\
\hline 16 & DBU-BZA-1:1 & 1806.60 & 486.75 & 187.96 & 60.13 \\
\hline 17 & DBU-PCA-2:1 & 376.71 & 136.80 & 61.97 & 32.86 \\
\hline
\end{tabular}

aStandard uncertainties $u$ were $u(\eta)=0.06$ and $u(\mathrm{~T})=0.03 \mathrm{~K}$. "--" represents that the EML was solid at ambient temperature, and the corresponding viscosity was not determined. 
Table S3. The density $(\rho)$ of the prepared EMLs at different temperatures.

\begin{tabular}{|c|c|c|c|c|c|}
\hline \multirow{2}{*}{ Entry } & \multirow{2}{*}{ EML } & \multicolumn{4}{|c|}{ Density $\left(\mathrm{g} \mathrm{cm}^{-3}\right)$} \\
\hline & & $25^{\circ} \mathrm{C}$ & $35^{\circ} \mathrm{C}$ & $45^{\circ} \mathrm{C}$ & $55^{\circ} \mathrm{C}$ \\
\hline 1 & DBU-LA-2:1 & 1.0652 & 1.0588 & 1.0539 & 1.0485 \\
\hline 2 & DBU-LA-1:1 & 1.1287 & 1.1218 & 1.1149 & 1.1108 \\
\hline 3 & DBU-LA-1:2 & 1.1524 & 1.1455 & 1.1386 & 1.1319 \\
\hline 4 & DBU-LA-1:3 & 1.1703 & 1.1629 & 1.1554 & 1.1478 \\
\hline 5 & DBU-PEG-2:1 & 1.0580 & 1.0503 & 1.0427 & 1.0351 \\
\hline 6 & DBU-PEG-1:1 & 1.0758 & 1.0681 & 1.0604 & 1.0527 \\
\hline 7 & DBU-PEG-1:2 & 1.0917 & 1.0839 & 1.0761 & 1.0683 \\
\hline 8 & DBU-PEG-1:3 & 1.0989 & 1.0910 & 1.0831 & 1.0725 \\
\hline 9 & DBU-CPL-2:1 & 1.0343 & 1.0154 & 1.0133 & 1.0036 \\
\hline 10 & DBU-CPL-1:1 & 1.0347 & 1.0271 & 1.0195 & 1.0118 \\
\hline 11 & DBU-CPL-1:2 & -- & -- & -- & -- \\
\hline 12 & DBU-CPL-1:3 & -- & -- & -- & -- \\
\hline 13 & DBU-NMTU-2:1 & 1.0451 & 1.0387 & 1.0336 & 1.0282 \\
\hline 14 & DBU-NMTU-1:1 & 1.0958 & 1.0885 & 1.0808 & 1.0735 \\
\hline 15 & DBU-BZA-2:1 & 1.0569 & 1.0499 & 1.0444 & 1.0384 \\
\hline 16 & DBU-BZA-1:1 & 1.0880 & 1.0759 & 1.0441 & 1.0353 \\
\hline 17 & DBU-PCA-2:1 & 1.0473 & 1.0411 & 1.0353 & 1.0297 \\
\hline \multicolumn{6}{|c|}{$\begin{array}{l}\text { aStandard uncertainties } u \text { were } u(\rho)=5 \times 10^{-4} \mathrm{~g} / \mathrm{m}^{-3} \text { and } u(\mathrm{~T})=0.01 \mathrm{~K} \text {. "--" represents tha } \\
\text { the EML was solid at ambient temperature, and the corresponding density was no } \\
\text { determined. }\end{array}$} \\
\hline
\end{tabular}


Table S4. Solubility of DAL at different temperatures.

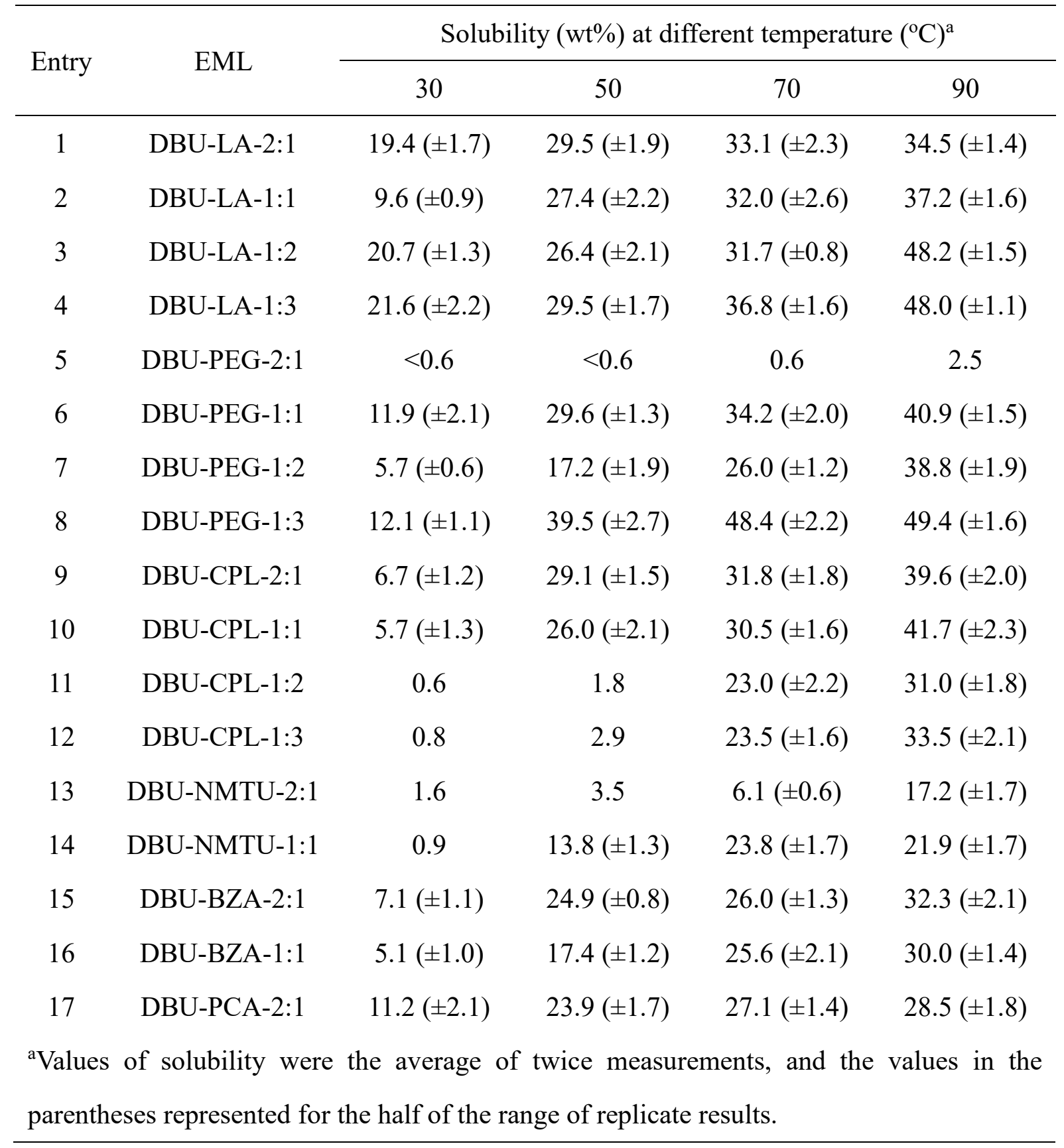


Table S5. Solubility of EHL at different temperatures.

\begin{tabular}{|c|c|c|c|c|c|}
\hline \multirow{2}{*}{ Entry } & \multirow{2}{*}{ EML } & \multicolumn{4}{|c|}{ Solubility $(w t \%)$ at different temperature $\left({ }^{\circ} \mathrm{C}\right)^{\mathrm{a}}$} \\
\hline & & 30 & 50 & 70 & 90 \\
\hline 1 & DBU-LA-2:1 & 4.7 & $13.6( \pm 1.7)$ & $24.6( \pm 1.6)$ & $26.9( \pm 1.3)$ \\
\hline 2 & DBU-LA-1:1 & 0.6 & 2.3 & 4.1 & $18.3( \pm 1.4)$ \\
\hline 3 & DBU-LA-1:2 & 0.8 & 4.3 & $11.9( \pm 1.7)$ & $21.7( \pm 0.9)$ \\
\hline 4 & DBU-LA-1:3 & $5.2( \pm 1.1)$ & $20.0( \pm 2.2)$ & $34.0( \pm 1.7)$ & $35.3( \pm 1.2)$ \\
\hline 5 & DBU-PEG-2:1 & $<2.2$ & $<2.2$ & $<2.2$ & 2.2 \\
\hline 6 & DBU-PEG-1:1 & 2.8 & $8.8( \pm 1.4)$ & $25.1( \pm 1.7)$ & $38.8( \pm 1.6)$ \\
\hline 7 & DBU-PEG-1:2 & 1.3 & $5.5( \pm 1.1)$ & $23.8( \pm 1.7)$ & $42.1( \pm 2.2)$ \\
\hline 8 & DBU-PEG-1:3 & 1.1 & $26.0( \pm 1.5)$ & $39.9( \pm 1.8)$ & $41.1( \pm 1.0)$ \\
\hline 9 & DBU-CPL-2:1 & $<1.4$ & $<1.4$ & $<1.4$ & 1.4 \\
\hline 10 & DBU-CPL-1:1 & $<0.6$ & $<0.6$ & $<0.6$ & 0.6 \\
\hline 11 & DBU-CPL-1:2 & $<0.9$ & $<0.9$ & $<0.9$ & 0.9 \\
\hline 12 & DBU-CPL-1:3 & $<3.1$ & $<3.1$ & $<3.1$ & 3.1 \\
\hline 13 & $\begin{array}{c}\text { DBU-NMTU- } \\
2: 1\end{array}$ & 1.2 & $5.1( \pm 0.7)$ & $23.1( \pm 1.3)$ & $32.7( \pm 2.4)$ \\
\hline 14 & $\begin{array}{c}\text { DBU-NMTU- } \\
1: 1\end{array}$ & $<0.2$ & 0.8 & $17.5( \pm 1.9)$ & $24.7( \pm 1.5)$ \\
\hline 15 & DBU-BZA-2:1 & 1.2 & $7.9( \pm 1.7)$ & $15.2( \pm 2.1)$ & $23.6( \pm 1.9)$ \\
\hline 16 & DBU-BZA-1:1 & $<0.4$ & 0.4 & 1.6 & $15.4( \pm 1.6)$ \\
\hline 17 & DBU-PCA-2:1 & 1.6 & $5.8( \pm 0.7)$ & $20.1( \pm 1.1)$ & $28.8( \pm 2.0)$ \\
\hline
\end{tabular}


Table S6. Solubility (wt $\%$ ) of OL at different temperatures.

\begin{tabular}{|c|c|c|c|c|c|}
\hline \multirow{2}{*}{ Entry } & \multirow{2}{*}{ EML } & \multicolumn{4}{|c|}{ Solubility at different temperature $\left({ }^{\circ} \mathrm{C}\right)^{\mathrm{a}}$} \\
\hline & & 30 & 50 & 70 & 90 \\
\hline 1 & DBU-LA-2:1 & $6.0( \pm 0.5)$ & $10.3( \pm 1.4)$ & $19.1( \pm 1.9)$ & $21.9( \pm 1.4)$ \\
\hline 2 & DBU-LA-1:1 & -- & -- & 1.1 & 3.9 \\
\hline 3 & DBU-LA-1:2 & $5.1( \pm 1.3)$ & $18.6( \pm 0.9)$ & $24.9( \pm 2.1)$ & $31.8( \pm 1.8)$ \\
\hline 4 & DBU-LA-1:3 & $12.8( \pm 1.1)$ & $30.6( \pm 1.6)$ & $35.1( \pm 1.4)$ & $37.3( \pm 1.6)$ \\
\hline 5 & DBU-PEG-2:1 & -- & -- & -- & -- \\
\hline 6 & DBU-PEG-1:1 & -- & -- & -- & -- \\
\hline 7 & DBU-PEG-1:2 & $<1.7$ & $<1.7$ & 1.7 & $23.8( \pm 1.1)$ \\
\hline 8 & DBU-PEG-1:3 & $<1.1$ & $<1.1$ & 1.1 & $11.4( \pm 1.6)$ \\
\hline 9 & DBU-CPL-2:1 & -- & -- & -- & -- \\
\hline 10 & DBU-CPL-1:1 & -- & -- & -- & -- \\
\hline 11 & DBU-CPL-1:2 & -- & -- & -- & -- \\
\hline 12 & DBU-CPL-1:3 & -- & -- & -- & -- \\
\hline 13 & DBU-NMTU-2:1 & $<1.6$ & 1.6 & $7.1( \pm 1.4)$ & $19.7( \pm 2.0)$ \\
\hline 14 & DBU-NMTU-1:1 & $<2.3$ & $<2.3$ & 2.3 & $18.7( \pm 1.7)$ \\
\hline 15 & DBU-BZA-2:1 & $<1.1$ & 1.1 & $5.8( \pm 1.5)$ & $12.7( \pm 1.4)$ \\
\hline 16 & DBU-BZA-1:1 & $<1.8$ & $<1.8$ & 1.8 & $22.1( \pm 2.6)$ \\
\hline 17 & DBU-PCA-2:1 & $<1.1$ & 1.1 & $25.7( \pm 1.1)$ & $30.6( \pm 1.9)$ \\
\hline \multicolumn{6}{|c|}{$\begin{array}{l}\text { a'Values of solubility were the average of twice measurements, and the values in the } \\
\text { parentheses represented for the half of the range of replicate results. "--" represents that the }\end{array}$} \\
\hline
\end{tabular}


Table S7. Solubility of SL at different temperatures.

\begin{tabular}{|c|c|c|c|c|c|}
\hline \multirow{2}{*}{ Entry } & \multirow{2}{*}{ EML } & \multicolumn{4}{|c|}{ Solubility $(w t \%)$ at different temperature $\left({ }^{\circ} \mathrm{C}\right)^{\mathrm{a}}$} \\
\hline & & 30 & 50 & 70 & 90 \\
\hline 1 & DBU-LA-2:1 & 0.8 & $5.6( \pm 1.2)$ & $23.1( \pm 0.7)$ & $32.0( \pm 1.7)$ \\
\hline 2 & DBU-LA-1:1 & 1.3 & $7.0( \pm 0.9)$ & $28.7( \pm 1.8)$ & $35.5( \pm 2.1)$ \\
\hline 3 & DBU-LA-1:2 & $5.0( \pm 0.6)$ & $18.0( \pm 1.8)$ & $35.7( \pm 1.5)$ & $37.2( \pm 1.4)$ \\
\hline 4 & DBU-LA-1:3 & $12.0( \pm 1.2)$ & $23.4( \pm 2.3)$ & $37.0( \pm 1.3)$ & $38.3( \pm 1.9)$ \\
\hline 5 & DBU-PEG-2:1 & $<2.3$ & $<2.3$ & $<2.3$ & 2.3 \\
\hline 6 & DBU-PEG-1:1 & $<1.3$ & $<1.3$ & $<1.3$ & 1.3 \\
\hline 7 & DBU-PEG-1:2 & $<0.9$ & $<0.9$ & $<0.9$ & 0.9 \\
\hline 8 & DBU-PEG-1:3 & $<0.9$ & $<0.9$ & $<0.9$ & 0.9 \\
\hline 9 & DBU-CPL-2:1 & $<1.4$ & $<1.4$ & $<1.4$ & 1.4 \\
\hline 10 & DBU-CPL-1:1 & $<0.7$ & $<0.7$ & $<0.7$ & 0.7 \\
\hline 11 & DBU-CPL-1:2 & $<1.2$ & $<1.2$ & $<1.2$ & 1.2 \\
\hline 12 & DBU-CPL-1:3 & $<3.1$ & $<3.1$ & $<3.1$ & 3.1 \\
\hline \multirow[t]{2}{*}{13} & DBU-NMTU- & $<1.7$ & $<1.7$ & $<1.7$ & 1.7 \\
\hline & $2: 1$ & & & & \\
\hline \multirow[t]{2}{*}{14} & DBU-NMTU- & $<0.8$ & $<0.8$ & $<0.8$ & 0.8 \\
\hline & $1: 1$ & & & & \\
\hline 15 & DBU-BZA-2:1 & $<1.2$ & $<1.2$ & $<1.2$ & 1.2 \\
\hline 16 & DBU-BZA-1:1 & $<0.8$ & $<0.8$ & $<0.8$ & 0.8 \\
\hline 17 & DBU-PCA-2:1 & $<1.9$ & $<1.9$ & $<1.9$ & 1.9 \\
\hline $\begin{array}{l}{ }^{a} \text { Value } \\
\text { parent }\end{array}$ & $\begin{array}{l}\text { of solubility } \\
\text { eses represented }\end{array}$ & ere the ave & of twice $m$ & $\begin{array}{l}\text { ements, an } \\
\text { e results. }\end{array}$ & e values in \\
\hline
\end{tabular}


Table S8. The solubility of lignin in different solvent from other researches

\begin{tabular}{|c|c|c|c|c|c|}
\hline Entry & Solution & $\mathrm{T}_{\text {test }}\left({ }^{\circ} \mathrm{C}\right)$ & Lignin & Solubility & Ref \\
\hline 1 & $\begin{array}{l}\text { propionic acid- Urea- } \\
\qquad 2: 1\end{array}$ & 40 & $\mathrm{OL}$ & $2 \mathrm{wt} \%$ & 4 \\
\hline 2 & $\begin{array}{l}\text { propionic acid- Urea- } \\
\qquad 2: 1\end{array}$ & 50 & $\mathrm{OL}$ & $4 \mathrm{wt} \%$ & 4 \\
\hline 3 & $\begin{array}{l}\text { propionic acid- Urea- } \\
\qquad 2: 1\end{array}$ & 40 & Kraft lignin & $25 \mathrm{wt} \%$ & 5 \\
\hline 4 & $\begin{array}{l}\text { propionic acid- Urea- } \\
\qquad 2: 1\end{array}$ & 50 & Kraft lignin & $15 w t \%$ & 5 \\
\hline 5 & $\begin{array}{l}\text { Lactic acid-Betaine- } \\
\qquad 2: 1\end{array}$ & 60 & $\begin{array}{c}\text { Alkali lignin, } \\
\text { (low sulfonate content) }\end{array}$ & $12.03 \mathrm{wt} \%$ & 5 \\
\hline 6 & $\begin{array}{c}\text { Malic acid- Proline- } \\
1: 3\end{array}$ & 100 & $\begin{array}{c}\text { Alkali lignin, } \\
\text { (low sulfonate content) }\end{array}$ & $14.9 \mathrm{wt} \%$ & 5 \\
\hline 7 & {$[\mathrm{C} 2 \mathrm{C} 1 \mathrm{im}][\mathrm{MeCO} 2]$} & 90 & $\begin{array}{c}\text { Kraft lignin } \\
(\text { Indulin AT) }\end{array}$ & $30 \mathrm{wt} \%$ & 6 \\
\hline 8 & [C4C1im][MeSO4] & 50 & $\begin{array}{c}\text { Residual } \\
\text { softwood } \\
\text { lignin }\end{array}$ & $26 \mathrm{wt} \%$ & 7 \\
\hline 9 & [P4441][MeSO4] & 90 & Kraft lignin & $\begin{array}{l}600 \pm 15 \\
\mathrm{~g} / \mathrm{kg}\end{array}$ & 8 \\
\hline 10 & {$[\mathrm{BMPyr}][\mathrm{N}(\mathrm{CN}) 2]$} & 90 & Kraft lignin & $\begin{array}{c}390 \pm 10 \\
\mathrm{~g} / \mathrm{kg}\end{array}$ & 8 \\
\hline 11 & {$[\mathrm{P} 44416][\mathrm{Cl}]$} & 90 & Kraft lignin & $\begin{array}{c}330 \pm 15 \\
\mathrm{~g} / \mathrm{kg}\end{array}$ & 8 \\
\hline 12 & {$[\mathrm{BMPyr}][\mathrm{N}(\mathrm{CN}) 2]$} & 90 & lignin sulfonate & $\begin{array}{c}560 \pm 15 \\
\mathrm{~g} / \mathrm{kg}\end{array}$ & 8 \\
\hline 13 & [Chol][OAc] & 90 & lignin sulfonate & $\begin{array}{c}330 \pm 10 \\
\mathrm{~g} / \mathrm{kg}\end{array}$ & 8 \\
\hline
\end{tabular}


Table S9. The solvatochromic parameters of the prepared EMLs. ${ }^{\text {a }}$

\begin{tabular}{|c|c|c|c|c|}
\hline Entry & EML & $\pi^{*}$ & $\alpha$ & $\beta$ \\
\hline 1 & DBU-LA-2:1 & $0.594( \pm 0.002)$ & $0.805( \pm 0.004)$ & $-0.935( \pm 0.002)$ \\
\hline 2 & DBU-LA-1:1 & $0.615( \pm 0.003)$ & $0.832( \pm 0.002)$ & $-0.809( \pm 0.005)$ \\
\hline 3 & DBU-LA-1:2 & $0.630( \pm 0.005)$ & $0.850( \pm 0.003)$ & $-0.833( \pm 0.004)$ \\
\hline 4 & DBU-LA-1:3 & $0.675( \pm 0.003)$ & $0.905( \pm 0.002)$ & $-0.862( \pm 0.003)$ \\
\hline 5 & DBU-PEG-2:1 & $0.514( \pm 0.002)$ & $0.707( \pm 0.002)$ & $1.019( \pm 0.004)$ \\
\hline 6 & DBU-PEG-1:1 & $0.530( \pm 0.003)$ & $0.727( \pm 0.005)$ & $0.909( \pm 0.003)$ \\
\hline 7 & DBU-PEG-1:2 & $0.552( \pm 0.004)$ & $0.692( \pm 0.002)$ & $0.900( \pm 0.003)$ \\
\hline 8 & DBU-PEG-1:3 & $0.563( \pm 0.002)$ & $0.767( \pm 0.003)$ & $0.918( \pm 0.005)$ \\
\hline 9 & DBU-CPL-2:1 & $0.496( \pm 0.002)$ & $0.685( \pm 0.004)$ & $-0.943( \pm 0.006)$ \\
\hline 10 & DBU-CPL-1:1 & $0.545( \pm 0.003)$ & $0.746( \pm 0.003)$ & $1.149( \pm 0.005)$ \\
\hline 11 & DBU-CPL-1:2 & $0.530( \pm 0.004)$ & $0.702( \pm 0.003)$ & $-0.683( \pm 0.004)$ \\
\hline 12 & DBU-CPL-1:3 & $0.527( \pm 0.005)$ & $0.724( \pm 0.002)$ & $1.045( \pm 0.005)$ \\
\hline 13 & DBU-NMTU-2:1 & $0.587( \pm 0.003)$ & $0.797( \pm 0.003)$ & $-0.979( \pm 0.002)$ \\
\hline 14 & DBU-NMTU-1:1 & $0.665( \pm 0.006)$ & $0.898( \pm 0.002)$ & $-0.879( \pm 0.004)$ \\
\hline 15 & DBU-BZA-2:1 & $0.580( \pm 0.004)$ & $0.789( \pm 0.003)$ & $1.399( \pm 0.005)$ \\
\hline 16 & DBU-BZA-1:1 & $0.583( \pm 0.005)$ & $1.273( \pm 0.002)$ & $1.374( \pm 0.004)$ \\
\hline 17 & DBU-PCA-2:1 & $0.539( \pm 0.003)$ & $0.738( \pm 0.004)$ & $-0.197( \pm 0.002)$ \\
\hline \multicolumn{5}{|c|}{$\begin{array}{l}\text { aThe value of the corresponding solvatochromic parameters was the average of twice } \\
\text { measurements, and the values in the parentheses were the half of the range of replicate }\end{array}$} \\
\hline
\end{tabular}


Table S10. The solubility of DAL in DBU-CPL-X and the solvatochromic parameters of DBU-CPL-X. ${ }^{a}$

$$
\text { Solubility (wt } \%) \text { at different }
$$

\begin{tabular}{|c|c|c|c|c|c|c|c|c|c|}
\hline \multirow[t]{2}{*}{ Entry } & \multirow[t]{2}{*}{ EML } & \multicolumn{4}{|c|}{ temperature $\left({ }^{\circ} \mathrm{C}\right)$} & \multirow[t]{2}{*}{$\pi^{*}$} & \multirow[t]{2}{*}{$\alpha$} & \multirow[t]{2}{*}{$\beta$} & \multirow[t]{2}{*}{$\alpha-\beta$} \\
\hline & & 30 & 50 & 70 & 90 & & & & \\
\hline \multirow{3}{*}{1} & DBU- & 6.7 & 29.1 & 31.8 & 39.6 & 0.496 & 0.685 & -0.943 & 1.628 \\
\hline & CPL- & $( \pm 1.2)$ & $( \pm 1.5)$ & $( \pm 1.8)$ & $( \pm 2.0)$ & $( \pm 0.002)$ & $( \pm 0.004)$ & $( \pm 0.006)$ & \\
\hline & $2: 1$ & & & & & & & & \\
\hline \multirow{3}{*}{2} & DBU- & 5.7 & 26.0 & 30.5 & 41.7 & 0.545 & 0.746 & 1.149 & \\
\hline & CPL- & $( \pm 1.3)$ & $( \pm 2.1)$ & $( \pm 1.6)$ & $( \pm 2.3)$ & $( \pm 0.003)$ & $( \pm 0.003)$ & $( \pm 0.005)$ & \\
\hline & $1: 1$ & & & & & & & & -0.403 \\
\hline \multirow{3}{*}{3} & DBU- & 0.6 & 1.8 & 23.0 & 31.0 & 0.530 & 0.702 & -0.683 & \\
\hline & CPL- & & & $( \pm 2.2)$ & $( \pm 1.8)$ & $( \pm 0.004)$ & $( \pm 0.003)$ & $( \pm 0.004)$ & \\
\hline & $1: 2$ & & & & & & & & 1.385 \\
\hline \multirow{3}{*}{4} & DBU- & 0.7 & 2.9 & 23.5 & 33.5 & 0.527 & 0.724 & 1.045 & \\
\hline & CPL- & & & $( \pm 1.6)$ & $( \pm 2.1)$ & $( \pm 0.005)$ & $( \pm 0.002)$ & $( \pm 0.005)$ & \\
\hline & $1: 3$ & & & & & & & & -0.321 \\
\hline
\end{tabular}

${ }^{a}$ Values of solubility and the corresponding solvatochromic parameters were the average of twice measurements, and the values in the parentheses represented for the half of the range of replicate results. 
Table S11. The solubility of DAL in DBU-NMTU-x and the solvatochromic parameters of DBU-NMTU-x. ${ }^{a}$

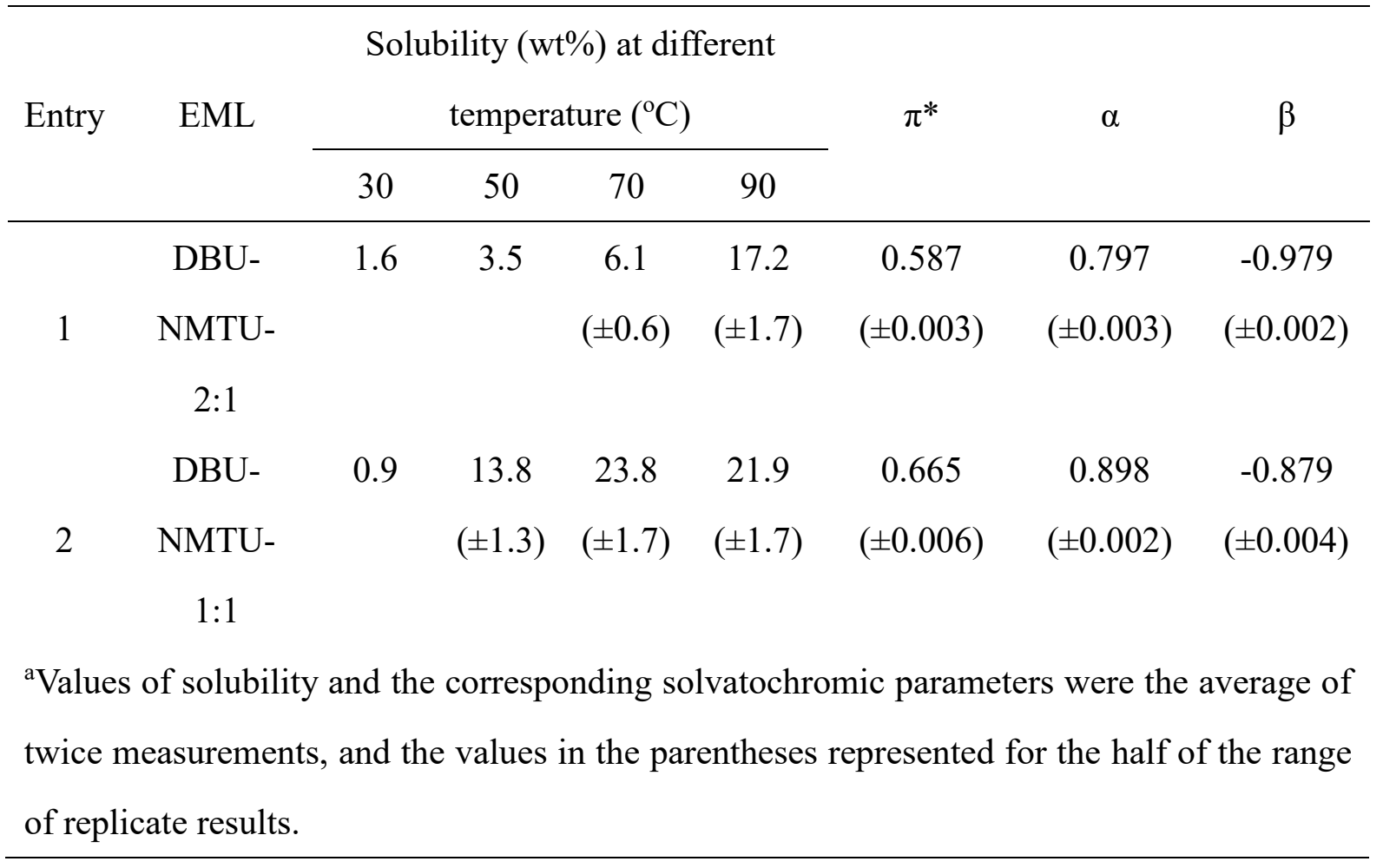


Table S12. The solubility of DAL in DBU-BZA-x and the solvatochromic parameters of DBUBZA-X. ${ }^{\text {a }}$

\begin{tabular}{|c|c|c|c|c|c|c|c|c|}
\hline \multirow{3}{*}{ Entry } & \multirow{3}{*}{ EML } & \multicolumn{5}{|c|}{ Solubility (wt $\%$ ) at different } & \multirow{3}{*}{$\alpha$} & \multirow{3}{*}{$\beta$} \\
\hline & & & temper: & ture $\left({ }^{\circ} \mathrm{C}\right.$ & & $\pi^{*}$ & & \\
\hline & & 30 & 50 & 70 & 90 & & & \\
\hline \multirow{2}{*}{1} & DBU- & 7.1 & 24.9 & 26.0 & 32.3 & 0.580 & 0.789 & 1.399 \\
\hline & BZA-2:1 & $( \pm 1.1)$ & $( \pm 0.8)$ & $( \pm 1.3)$ & $( \pm 2.1)$ & $( \pm 0.004)$ & $( \pm 0.003)$ & $( \pm 0.005)$ \\
\hline \multirow{2}{*}{2} & DBU- & 5.1 & 17.4 & 25.6 & 30.0 & 0.583 & 1.273 & 1.374 \\
\hline & BZA-1:1 & $( \pm 1.0)$ & $( \pm 1.2)$ & $( \pm 2.1)$ & $( \pm 1.4)$ & $( \pm 0.005)$ & $( \pm 0.002)$ & $( \pm 0.004)$ \\
\hline \multicolumn{9}{|c|}{${ }^{a}$ Values of solubility and the corresponding solvatochromic parameters were the average of } \\
\hline \multicolumn{9}{|c|}{ twice measurements, and the values in the parentheses represented for the half of the range } \\
\hline \multicolumn{9}{|c|}{ of replicate results. } \\
\hline
\end{tabular}


Table S13. The solubility of EHL in DBU-PEG-x and the solvatochromic parameters of DBUPEG-x..$^{\mathrm{a}}$

\begin{tabular}{|c|c|c|c|c|c|c|c|c|}
\hline \multirow{3}{*}{ Entry } & \multirow{3}{*}{ EML } & \multicolumn{4}{|c|}{ Solubility (wt $\%$ ) at different } & \multirow{3}{*}{$\pi^{*}$} & \multirow{3}{*}{$\alpha$} & \multirow{3}{*}{$\beta$} \\
\hline & & \multicolumn{4}{|c|}{ temperature $\left({ }^{\circ} \mathrm{C}\right)$} & & & \\
\hline & & 30 & 50 & 70 & 90 & & & \\
\hline 1 & $\begin{array}{c}\text { DBU- } \\
\text { PEG-2:1 }\end{array}$ & $<2.2$ & $<2.2$ & $<2.2$ & 2.2 & $\begin{array}{c}0.514 \\
( \pm 0.002)\end{array}$ & $\begin{array}{c}0.707 \\
( \pm 0.002)\end{array}$ & $\begin{array}{c}1.019 \\
( \pm 0.004)\end{array}$ \\
\hline 2 & $\begin{array}{c}\text { DBU- } \\
\text { PEG-1:1 }\end{array}$ & 2.8 & $\begin{array}{c}8.8 \\
( \pm 1.4)\end{array}$ & $\begin{array}{c}25.1 \\
( \pm 1.7)\end{array}$ & $\begin{array}{c}38.8 \\
( \pm 1.6)\end{array}$ & $\begin{array}{c}0.530 \\
( \pm 0.003)\end{array}$ & $\begin{array}{c}0.727 \\
( \pm 0.005)\end{array}$ & $\begin{array}{c}0.909 \\
( \pm 0.003)\end{array}$ \\
\hline 3 & $\begin{array}{c}\text { DBU- } \\
\text { PEG-1:2 }\end{array}$ & 1.3 & $\begin{array}{c}5.5 \\
( \pm 1.1)\end{array}$ & $\begin{array}{r}23.8 \\
( \pm 1.7)\end{array}$ & $\begin{array}{c}42.1 \\
( \pm 2.2)\end{array}$ & $\begin{array}{c}0.552 \\
( \pm 0.004)\end{array}$ & $\begin{array}{c}0.692 \\
( \pm 0.002)\end{array}$ & $\begin{array}{c}0.900 \\
( \pm 0.003)\end{array}$ \\
\hline 4 & $\begin{array}{c}\text { DBU- } \\
\text { PEG-1:3 }\end{array}$ & 1.1 & $\begin{array}{c}26.0 \\
( \pm 1.5)\end{array}$ & $\begin{array}{r}39.9 \\
( \pm 1.8)\end{array}$ & $\begin{array}{c}41.1 \\
( \pm 1.0)\end{array}$ & $\begin{array}{c}0.563 \\
( \pm 0.002)\end{array}$ & $\begin{array}{c}0.767 \\
( \pm 0.003)\end{array}$ & $\begin{array}{c}0.918 \\
( \pm 0.005)\end{array}$ \\
\hline $\begin{array}{l}{ }^{a} \text { Values } \\
\text { twice } \mathrm{r} \\
\text { of repl }\end{array}$ & $\begin{array}{l}\text { of solubilit } \\
\text { easurement } \\
\text { cate results. }\end{array}$ & and th & values & $\begin{array}{l}\text { nding } s \\
n \text { the } p\end{array}$ & $\begin{array}{l}\text { vatochr } \\
\text { entheses }\end{array}$ & $\begin{array}{l}\text { ic param } \\
\text { presented }\end{array}$ & $\begin{array}{l}\text { os were the } \\
\text { the half }\end{array}$ & $\begin{array}{l}\text { Iverage of } \\
\text { the range }\end{array}$ \\
\hline
\end{tabular}


Table S14. The solubility of EHL in DBU-BZA-x and DBU-NMTU-x, and the solvatochromic parameters of DBU-BZA-x and DBU-NMTU-x. ${ }^{a}$

Solubility (wt $\%)$ at different

\begin{tabular}{|c|c|c|c|c|c|c|c|c|}
\hline \multirow[t]{2}{*}{ Entry } & \multirow[t]{2}{*}{ EML } & \multicolumn{4}{|c|}{ temperature $\left({ }^{\circ} \mathrm{C}\right)$} & \multirow[t]{2}{*}{$\pi^{*}$} & \multirow[t]{2}{*}{$\alpha$} & \multirow[t]{2}{*}{$\beta$} \\
\hline & & 30 & 50 & 70 & 90 & & & \\
\hline 1 & $\begin{array}{c}\text { DBU- } \\
\text { BZA-2:1 }\end{array}$ & 1.2 & $\begin{array}{c}7.9 \\
( \pm 1.7)\end{array}$ & $\begin{array}{c}15.2 \\
( \pm 2.1)\end{array}$ & $\begin{array}{c}23.6 \\
( \pm 1.9)\end{array}$ & $\begin{array}{c}0.580 \\
( \pm 0.004)\end{array}$ & $\begin{array}{c}0.789 \\
( \pm 0.003)\end{array}$ & $\begin{array}{c}1.399 \\
( \pm 0.005)\end{array}$ \\
\hline 2 & $\begin{array}{c}\text { DBU- } \\
\text { BZA-1:1 }\end{array}$ & $<0.4$ & 0.4 & 1.6 & $\begin{array}{c}15.4 \\
( \pm 1.6)\end{array}$ & $\begin{array}{c}0.583 \\
( \pm 0.005)\end{array}$ & $\begin{array}{c}1.273 \\
( \pm 0.002)\end{array}$ & $\begin{array}{c}1.374 \\
( \pm 0.004)\end{array}$ \\
\hline 3 & $\begin{array}{c}\text { DBU- } \\
\text { NMTU- } \\
2: 1\end{array}$ & 1.2 & $\begin{array}{c}5.1 \\
( \pm 0.7)\end{array}$ & $\begin{array}{c}23.1 \\
( \pm 1.3)\end{array}$ & $\begin{array}{c}32.7 \\
( \pm 2.4)\end{array}$ & $\begin{array}{c}0.587 \\
( \pm 0.003)\end{array}$ & $\begin{array}{c}0.797 \\
( \pm 0.003)\end{array}$ & $\begin{array}{c}-0.979 \\
( \pm 0.002)\end{array}$ \\
\hline 4 & $\begin{array}{c}\text { DBU- } \\
\text { NMTU- } \\
1: 1\end{array}$ & $<0.2$ & 0.8 & $\begin{array}{c}17.5 \\
( \pm 1.9)\end{array}$ & $\begin{array}{r}24.7 \\
( \pm 1.5)\end{array}$ & $\begin{array}{c}0.665 \\
( \pm 0.006)\end{array}$ & $\begin{array}{c}0.898 \\
( \pm 0.002)\end{array}$ & $\begin{array}{c}-0.879 \\
( \pm 0.004)\end{array}$ \\
\hline $\begin{array}{l}\text { avalue } \\
\text { twice }\end{array}$ & $\begin{array}{l}\text { f solubility } \\
\text { asurement }\end{array}$ & and $t$ & value & the & atheses & $\begin{array}{l}\text { param } \\
\text { resented }\end{array}$ & the half & $\begin{array}{l}\text { Iverage of } \\
\text { the range }\end{array}$ \\
\hline
\end{tabular}



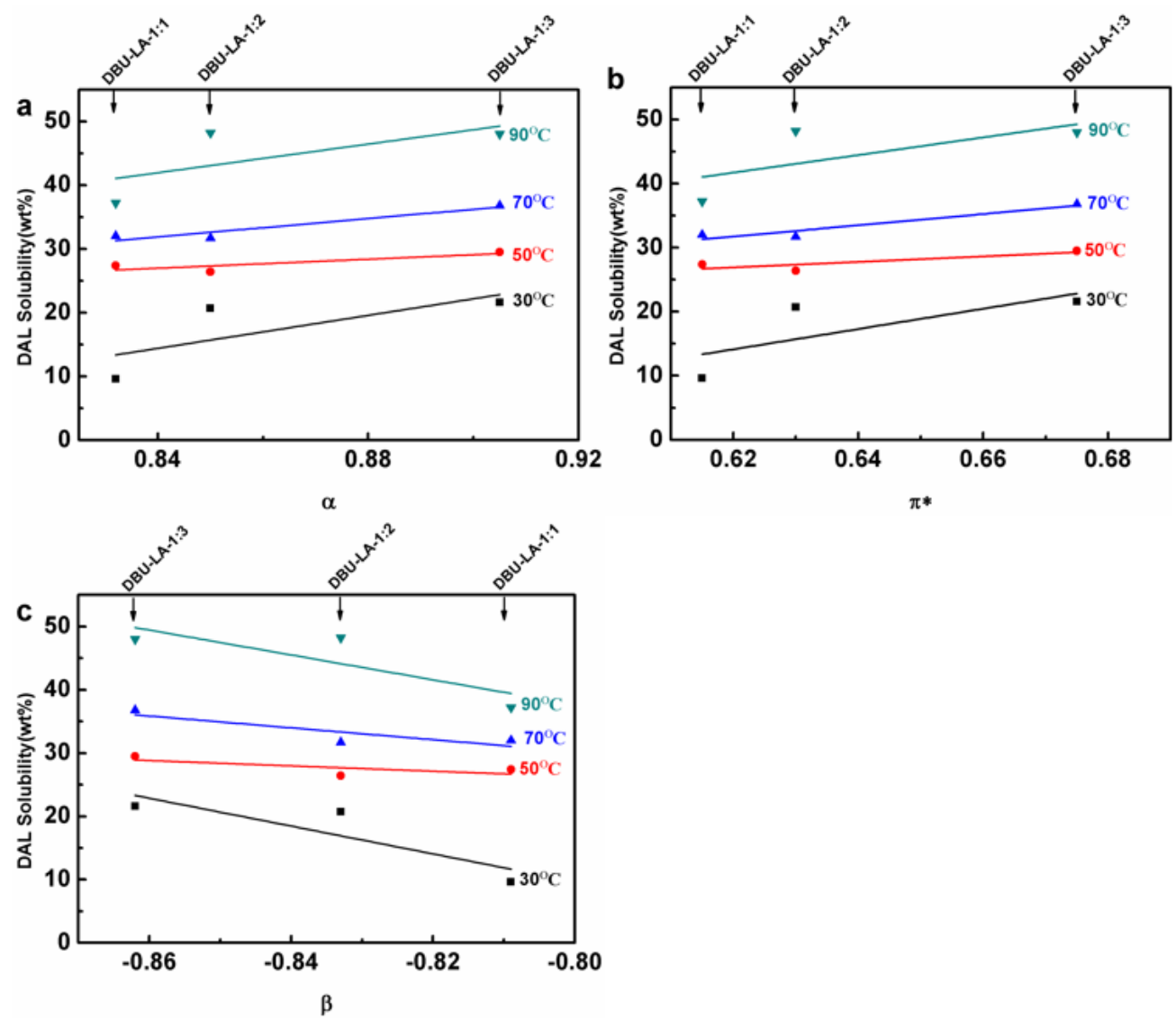

Fig. S1. The relationship between the DAL solubility in DBU-LA-x and the value of $\beta$ of DBULA-X when DBU-LA-2:1 was not considered. Additionally, it should be pointed out that values of solubility were the average of twice measurements, and the values of the solubility represented for the half of the range of replicate results. 

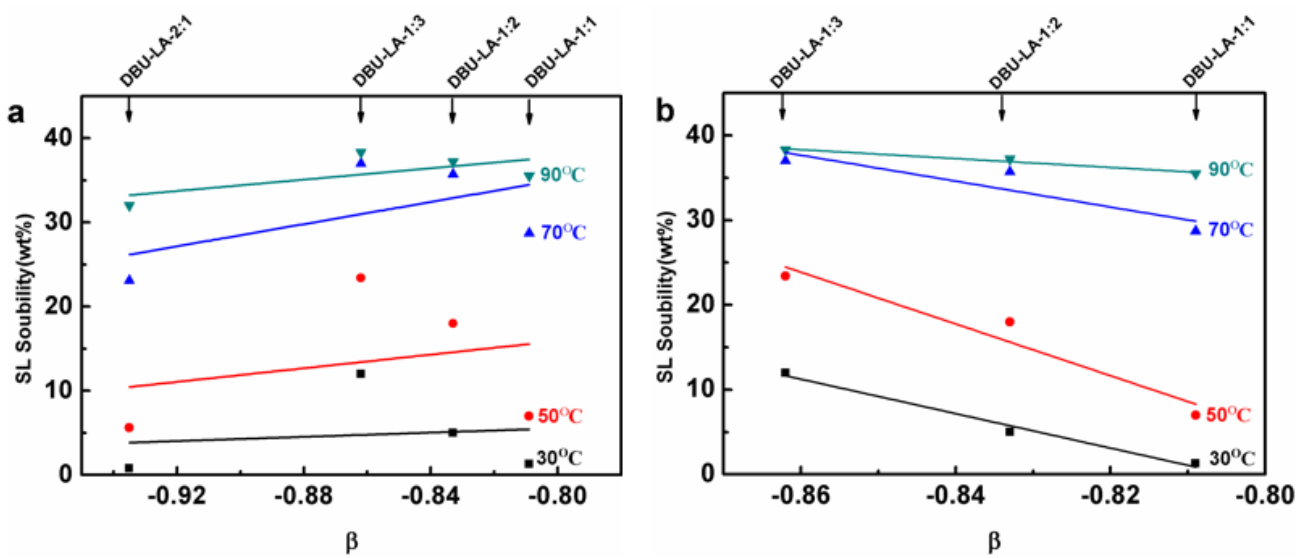

Fig. S2. The relationship between the SL solubility in DBU-LA-X and the value of $\beta$ of DBULA-x. (a) DBU-LA-2:1 was considered, and (b) DBU-LA-2:1 was excluded. Additionally, it should be pointed out that values of solubility were the average of twice measurements, and the values of the solubility represented for the half of the range of replicate results.

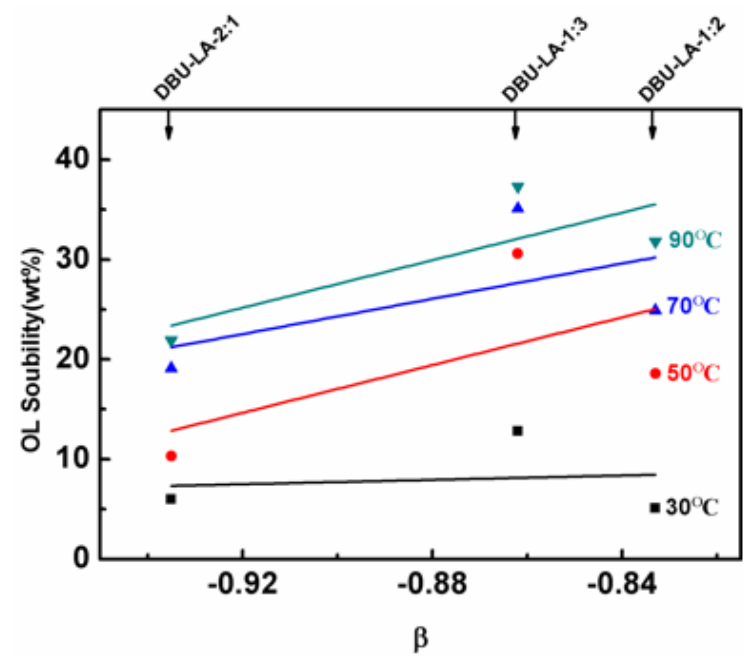

Fig. S3. The relationship between the OL solubility in DBU-LA-x and the value of $\beta$ of DBULA-x (DBU-LA-1:1 was not considered because of its low solubility of OL.). Additionally, it should be pointed out that values of solubility were the average of twice measurements, and the values of the solubility represented for the half of the range of replicate results. 


\section{REFERENCES}

1 R. Mistry, Characterisation and Applications of $\mathrm{CO}_{2}$-Expanded Solvents [D]. University of Leicester, 2008.

2 Y. Wu, T. Sasaki, K. Kazushi, T. Seo and K. Sakurai, Interactions between spiropyrans and room-temperature ionic liquids: photochromism and solvatochromism, J. Phys. Chem. B, 2008, 112, 7530 .

3 P. G. Jessop, D. A. Jessop, D. Fu and L. Phan, Solvatochromic parameters for solvents of interest in green chemistry, Green Chem., 2012, 14, 1245.

4 B. Soares, D. J. P. Tavares, J. L. Amaral, A. J. D. Silvestre, C. S. R. Freire and J. A. P. Coutinho, Enhanced Solubility of Lignin Monomeric Model Compounds and Technical Lignins in Aqueous Solutions of Deep Eutectic Solvents. ACS Sustainable Chem. Eng., 2017, $5(5), 4056$.

5 M. Francisco, A. van den Bruinhorst and M. C. Kroon, New natural and renewable low transition temperature mixtures (LTTMs): screening as solvents for lignocellulosic biomass processing. Green Chem., 2012, 14 (8), 2153.

6 D. Glas, C. Van Doorslaer, D. Depuydt, F. Liebner, T. Rosenau, K. Binnemans and D. E. De Vos, Lignin solubility in non-imidazolium ionic liquids. Journal of Chemical Technology \& Biotechnology, 2015, 90 (10), 1821.

7 Y. Q. Pu, N. Jiang and A. J. Ragauskas and J. Wood, Ionic Liquid as a Green Solvent for Lignin, Chem. Technol., 2007, 27, 23.

8 D. Fu, G. Mazza and Y. Tamaki and J. Agric, Lignin Extraction from Straw by Ionic Liquids and Enzymatic Hydrolysis of the Cellulosic Residues, Food Chem., 2010,58, 2915. 